\title{
Real Structured Singular Value Synthesis Using the Scaled Popov Criterion
}

\author{
Andrew G. Sparks* and Dennis S. Bernstein ${ }^{\dagger}$ \\ University of Michigan, Ann Arbor, Michigan 48109-2118
}

\begin{abstract}
The scaled Popov criterion is used to derive an upper bound for the worst-case $\boldsymbol{H}_{2}$ norm over a set of linear, time-invariant, norm-bounded, block-structured parameter perturbations. This upper bound provides the basis for a robust controller synthesis procedure that minimizes the upper bound and guarantees asymptotic stability of the closed-loop system for all real parameter perturbations in the specified set. Numerical examples demonstrate the tradeoff between achievable $\mathrm{H}_{2}$ performance and guaranteed robustness to real parameter perturbations as well as the reduction in conservatism due to the scaling matrix. Controllers designed for flexible structures using this technique are shown to have similarities to maximum entropy controllers designed for the same examples.
\end{abstract}

\begin{tabular}{ll}
\multicolumn{1}{c}{ Nomenclature } \\
$B_{0}, C_{0}=$ & fixed matrices characterizing the structure of \\
& uncertainty \\
$w(t)$ & $=$ zero-mean white noise with unit intensity \\
$x(t)$ & $=$ state vector \\
$z(t)$ & $=$ performance variables \\
$\Delta$ & $=$ set of block-structured matrices
\end{tabular}

\section{Introduction}

$\mathbf{T}$ IME-INVARIANT perturbations of a nominal plant can be modeled conservatively as complex, time-varying parameters or more precisely, although with greater difficulty, as real, timeinvariant parameters. ${ }^{1,2}$ The difference between a complex, timevarying model and a real, time-invariant model of time-invariant perturbations becomes evident in Lyapunov stability analysis. Using conventional Lyapunov functions, stability can be guaranteed for time-varying perturbations in the plant since the Lyapunov derivative need be negative only for each fixed value of time. When the perturbations are actually constant, however, modeling uncertain parameters as time-varying quantities leads to conservatism.

The positivity criterion, which guarantees stability for timevarying nonlinearities in the feedback loop, is based on a fixed Lyapunov function and, thus, is conservative in the case of timeinvariant nonlinearities. Alternatively, conservatism can be reduced by using parameter-dependent Lyapunov functions, where a family of Lyapunov functions depends on the constant uncertain real parameters. ${ }^{3}$ The multiplier in the Popov criterion, which is based on a parameter-dependent Lyapunov function, restricts the nonlinearities to be time invariant and, hence, yields less conservative results.

In recent work, the positivity and Popov criteria were specialized to the case of a nominal plant in a feedback interconnection with a linear perturbation representing parameter uncertainty. ${ }^{3,4}$ To further reduce conservatism, the multivariable Popov criterion was generalized to include scaling matrices. ${ }^{5}$ The resulting scaled Popov criterion yields a frequency domain upper bound for the structured singular value, ${ }^{6}$ which is shown to be equivalent to the mixed- $\mu$ upper bound of Fan et al. ${ }^{2}$ specialized to real parameter uncertainty. Then, using a generalization of the positive real lemma, the scaled Popov criterion is rewritten in state space form using linear matrix inequalities (LMIs).

Received Jan. 30, 1995; revision received April 17, 1995; accepted for publication April 24, 1995. This paper is declared a work of the U.S. Government and is not subject to copyright protection in the United States.

${ }^{*}$ Graduate Student, Department of Aerospace Engineering, 2020 FXB Building, 1320 Beal Street.

${ }^{\dagger}$ Associate Professor, Department of Aerospace Engineering, 3020 FXB Building, 1320 Beal Street.
The goal of the present paper is to apply the scaled Popov criterion ${ }^{5,6}$ to the problem of robust controller synthesis. Specifically, the state space form of the scaled Popov criterion is used to derive an upper bound for the worst-case $\mathrm{H}_{2}$ norm over the set of real parameter perturbations. Necessary conditions are derived for the control gains such that this upper bound for the worst-case $\mathrm{H}_{2}$ norm is minimized. The resulting controller guarantees that the closed-loop system is asymptotically stable and that the $\mathrm{H}_{2}$ norm of the closed-loop system is bounded by the optimum worst-case $\mathrm{H}_{2}$ norm for all real parameter perturbations in the specified set. The necessary conditions are then used with a quasi-Newton optimization algorithm to obtain robust controllers, and a numerical example is presented to demonstrate the reduction in conservatism due to the scaling matrix of the scaled Popov criterion. Finally, scaled Popov controllers are obtained for a flexible structure with collocated and noncollocated sensor/actuator pairs. These controllers are then compared with maximum entropy controllers obtained for the same examples.?

\section{Problem Statement}

Consider the linear, time-invariant uncertain system

$$
\begin{gathered}
\dot{x}(t)=\left(A+B_{0} \Delta C_{0}\right) x(t)+D w(t) \\
z(t)=E x(t)
\end{gathered}
$$

where $x(t) \in \mathbb{R}^{n}, w(t) \in \mathbb{R}^{d}, z(t) \in \mathbb{R}^{q}, B_{0} \in \mathbb{R}^{n \times m}$, and $C_{0} \in$ $\mathbb{R}^{m \times n}$. The uncertain matrix $\Delta$ is assumed to be an element of $\Delta_{\gamma}$, the set of real, norm-bounded perturbations defined by

$$
\Delta_{\gamma}=\left\{\Delta \in \Delta: \sigma_{\max }(\Delta) \leq \gamma^{-1}\right\}
$$

In Sec. IV, the model (1) and (2) will represent the interconnection of a plant and a controller, where the nominal dynamics matrix $A$ is asymptotically stable. For controller synthesis, consider the worstcase $\mathrm{H}_{2}$ norm from $w(t)$ to $z(t)$ given by

$$
J(\gamma)=\sup _{\Delta \in \Delta_{\gamma}}\left\|G_{\Delta}(s)\right\|_{2}^{2}
$$

where $G_{\Delta}(s)=E\left[s I-\left(A+B_{0} \Delta C_{0}\right)\right]^{-1} D$ and where $A+B_{0} \Delta C_{0}$ is assumed to be Hurwitz for all $\Delta \in \Delta_{\gamma}$. It follows from standard results that

$$
J(\gamma)=\sup _{\Delta \in \Delta_{Y}} \operatorname{tr} P_{\Delta} D D^{T}
$$

where $P_{\Delta}$ is the unique, nonnegative-definite solution to the Lyapunov equation

$$
0=\left(A+B_{0} \Delta C_{0}\right)^{T} P_{\Delta}+P_{\Delta}\left(A+B_{0} \Delta C_{0}\right)+E^{T} E
$$




\section{Scaled Popov Criterion}

In this section we review the scaled Popov criterion for normbounded, block-structured uncertainty. This criterion, which is given in the form of a Riccati equation, is then used to derive an upper bound for the worst-case $\mathrm{H}_{2}$ cost given by Eq. (3). We first give specific structure to the set $\Delta$ of block-structured perturbations by letting $\Delta$ denote the set of real, symmetric, block-diagonal matrices given by

$$
\begin{gathered}
\Delta=\left\{\Delta: \Delta=\text { block-diag }\left(I_{l_{1}} \otimes \Delta_{1}, \ldots, I_{l_{r}} \otimes \Delta_{r}\right)\right. \\
\left.\Delta_{i}=\Delta_{i}^{T} \in \mathbb{R}^{m_{i} \times m_{i}}, i=1, \ldots, r\right\}
\end{gathered}
$$

where the dimension $m_{i}$ and the number of repetitions $l_{i}$ of the uncertain block $\Delta_{i}$ are given. Note that $\sum_{i=1}^{r} m_{i} l_{i}=m$.

To state the scaled Popov criterion, define the set $\mathcal{N}$ of Hermitian matrices $N$ that commute with every matrix $\Delta \in \Delta$ by

$$
\begin{gathered}
\mathcal{N}=\left\{N: N=\text { block-diag }\left(N_{1} \otimes I_{m_{1}}, \ldots, N_{r} \otimes I_{m_{r}}\right)\right. \\
\left.N_{i}=N_{i}^{*} \in \mathbb{C}^{l_{i} \times l_{i}}, i=1, \ldots, r\right\}
\end{gathered}
$$

and the set $\mathcal{Q}$ of positive-definite matrices $Q$ that commute with every matrix $\Delta \in \Delta$ by

$$
\mathcal{Q}=\{Q \in \mathcal{N}: Q>0\}
$$

Note that if $\Delta \in \Delta, N \in \mathcal{N}$, and $Q \in \mathcal{Q}$, then $N \Delta=$ $\Delta N=$ block-diag $\left(N_{1} \otimes \Delta_{1}, \ldots, N_{r} \otimes \Delta_{r}\right)$ and $Q^{-1 / 2} \Delta Q^{1 / 2}=$ $\Delta Q^{-1 / 2} Q^{1 / 2}=\Delta$. Hence, for all $\Delta \in \Delta$ and $Q \in \mathcal{Q}$, the asymptotic stability of the feedback interconnection of $G(s)$ and $\Delta$ is equivalent to the asymptotic stability of the feedback interconnection of $Q^{1 / 2} G(s) Q^{-1 / 2}$ and $\Delta$.

We now state the scaled Popov criterion in the form of a Riccati equation and derive an upper bound for the cost Eq. (3)

Theorem 1: Let $\gamma>0$ and assume that $A-\gamma^{-1} B_{0} C_{0}$ is Hurwitz. If there exist positive-definite matrices $P$ and $R$ and matrices $N \in \mathcal{N}$ and $Q \in \mathcal{Q}$ such that $\gamma Q-N C_{0} B_{0}-B_{0}^{T} C_{0}^{T} N>0$ and

$$
\begin{aligned}
0 & =\left(A-\gamma^{-1} B_{0} C_{0}\right)^{T} P+P\left(A-\gamma^{-1} B_{0} C_{0}\right) \\
& +\left[B_{0}^{T} P+Q C_{0}+N C_{0}\left(A-\gamma^{-1} B_{0} C_{0}\right)\right]^{*} \\
& \times\left(\gamma Q-N C_{0} B_{0}-B_{0}^{T} C_{0}^{T} N\right)^{-1}\left(B_{0}^{T} P+Q C_{0}\right. \\
& \left.+N C_{0}\left(A-\gamma^{-1} B_{0} C_{0}\right)\right)+R
\end{aligned}
$$

then the feedback interconnection of $G(s)$ and $\Delta$ is asymptotically stable for all $\Delta \in \Delta_{\gamma}$. If, in addition, $R \geq E^{T} E$, then

$$
J(\gamma) \leq \operatorname{tr}\left(P+2 \gamma^{-1} C_{0}^{T} N C_{0}\right) D D^{T}
$$

\section{Robust Controller Synthesis}

We now use the upper bound for the $H_{2}$ cost given by Theorem 1 to synthesize robust controllers. Consider the $n$ th-order uncertain plant

$$
\begin{gathered}
\dot{x}(t)=\left(A+B_{0} \Delta C_{0}\right) x(t)+B u(t)+D_{1} w(t) \\
y(t)=C x(t)+D_{2} w(t) \\
z(t)=E_{1} x(t)+E_{2} u(t)
\end{gathered}
$$

where the $n_{c}$ th-order dynamic compensator, where $n_{c} \leq n$, is of the form

$$
\begin{gathered}
\dot{x}_{c}(t)=A_{c} x_{c}(t)+B_{c} y(t) \\
u(t)=C_{c} x_{c}(t)
\end{gathered}
$$

The closed-loop system can be written as

$$
\begin{gathered}
\dot{\tilde{x}}(t)=\left(\tilde{A}+\tilde{B}_{0} \Delta \tilde{C}_{0}\right) \tilde{x}(t)+\tilde{D} w(t) \\
z(t)=\tilde{E} \tilde{x}(t)
\end{gathered}
$$

where

$$
\begin{aligned}
\tilde{x}=\left[\begin{array}{c}
x(t) \\
x_{c}(t)
\end{array}\right], & \tilde{A}=\left[\begin{array}{cc}
A & B C_{c} \\
B_{c} C & A_{c}
\end{array}\right] \\
\tilde{B}_{0}=\left[\begin{array}{c}
B_{0} \\
0_{n_{c} \times m}
\end{array}\right], & \tilde{C}_{0}=\left[\begin{array}{ll}
C_{0} & 0_{m \times n_{c}}
\end{array}\right] \\
\tilde{D}=\left[\begin{array}{c}
D_{1} \\
B_{c} D_{2}
\end{array}\right], & \tilde{E}=\left[\begin{array}{ll}
E_{1} & E_{2} C_{c}
\end{array}\right]
\end{aligned}
$$

If $\tilde{A}+\tilde{B}_{0} \Delta \tilde{C}_{0}$ is Hurwitz for all $\Delta \in \Delta_{\gamma}$, then the closed-loop $H_{2}$ cost is

$$
J(\gamma)=\sup _{\Delta \in \Delta_{\gamma}} \operatorname{tr} \tilde{P}_{\Delta} \tilde{D} \tilde{D}^{T}
$$

where $\tilde{P}_{\Delta}$ is the unique, nonnegative-definite solution to the Lyapunov equation

$$
0=\left(\tilde{A}+\tilde{B}_{0} \Delta \tilde{C}_{0}\right)^{T} \tilde{P}_{\Delta}+\tilde{P}_{\Delta}\left(\tilde{A}+\tilde{B}_{0} \Delta \tilde{C}_{0}\right)+\tilde{E}^{T} \tilde{E}
$$

Now, applying Theorem 1 to the closed-loop system with $A=\tilde{A}$, $B_{0}=\widetilde{B}_{0}, C_{0}=\tilde{C}_{0}, E=\tilde{E}$, and $R=\tilde{E}^{T} \tilde{E}$, it follows that if $\tilde{A}-$ $\gamma^{-1} \tilde{B}_{0} \tilde{C}_{0}$ is Hurwitz and there exist a positive-definite matrix $\tilde{P}$ and matrices $N \in \mathcal{N}$ and $Q \in \mathcal{Q}$ such that $\gamma Q-N \tilde{C}_{0} \tilde{B}_{0}-\tilde{B}_{0}^{T} \tilde{C}_{0}^{T} N>0$ and

$$
\begin{aligned}
0 & =\left(\tilde{A}-\gamma^{-1} \tilde{B}_{0} \tilde{C}_{0}\right)^{T} \tilde{P}+\tilde{P}\left(\tilde{A}-\gamma^{-1} \tilde{B}_{0} \tilde{C}_{0}\right) \\
& +\left[\tilde{B}_{0}^{T} \tilde{P}+Q \tilde{C}_{0}+N \tilde{C}_{0}\left(\tilde{A}-\gamma^{-1} \tilde{B}_{0} \tilde{C}_{0}\right)\right]^{*} \\
& \times\left(\gamma Q-N \tilde{C}_{0} \tilde{B}_{0}-\tilde{B}_{0}^{T} \tilde{C}_{0}^{T} N\right)^{-1}\left[\tilde{B}_{0}^{T} \tilde{P}+Q \tilde{C}_{0}\right. \\
& \left.+N \tilde{C}_{0}\left(\tilde{A}-\gamma^{-1} \tilde{B}_{0} \tilde{C}_{0}\right)\right]+\tilde{E}^{T} \tilde{E}
\end{aligned}
$$

then the closed-loop system is asymptotically stable for all $\Delta \in \Delta_{\gamma}$ and

$$
J(\gamma) \leq \operatorname{tr}\left(\tilde{P}+2 \gamma^{-1} \tilde{C}_{0}^{T} N \tilde{C}_{0}\right) \tilde{D} \tilde{D}^{T}
$$

To synthesize robust controllers, we minimize this upper bound for the worst-case $\mathrm{H}_{2}$ cost. To formalize this approach, we define the auxiliary cost $\mathcal{J}\left(\gamma, A_{c}, B_{c}, C_{c}, N, Q\right)$ by

$$
\mathcal{J}\left(\gamma, A_{c}, B_{c}, C_{c}, N, Q\right)=\operatorname{tr}\left(\tilde{P}+2 \gamma^{-1} \tilde{C}_{0}^{T} N \tilde{C}_{0}\right) \tilde{D} \tilde{D}^{T}
$$

and pose the following auxiliary minimization problem:

Find controller matrices $A_{c}, B_{c}$, and $C_{c}$ and matrices $N \in \mathcal{N}$ and $Q \in \mathcal{Q}$ such that the auxiliary cost $\mathcal{J}\left(\gamma, A_{c}, B_{c}, C_{c}, N, Q\right)$ is minimized, where $\tilde{P}$ satisfies the Riccati equation (7) and $\gamma Q-$ $N \tilde{C}_{0} \tilde{B}_{0}-\tilde{B}_{0}^{T} \tilde{C}_{0}^{T} N>0$.

For convenience, partition the matrices $\tilde{P}$ and $\tilde{Q}$ as

$$
\tilde{P}=\left[\begin{array}{cc}
P_{1} & P_{12} \\
P_{12}^{T} & P_{2}
\end{array}\right], \quad \tilde{Q}=\left[\begin{array}{cc}
Q_{1} & Q_{12} \\
Q_{12}^{T} & Q_{2}
\end{array}\right]
$$

where $P_{1}, Q_{1} \in \mathbb{R}^{n \times n}$ and $P_{2}, Q_{2} \in \mathbb{R}^{n_{c} \times n_{c}}$. The following theorem provides gradients of the auxiliary $\operatorname{cost} \mathcal{J}\left(\gamma, A_{c}, B_{c}, C_{c}, N, Q\right)$ for controller synthesis.

Theorem 2: Suppose $A_{c}, B_{c}, C_{c}, N$, and $Q$ solve the auxiliary minimization problem. Then $\gamma Q-N \tilde{C}_{0} \tilde{B}_{0}-\tilde{B}_{0}^{T} \tilde{C}_{0}^{T} N>0$ and there exist positive-definite matrices $\tilde{P}$ and $\tilde{Q}$, where $\tilde{P}$ satisfies Eq. (7), $\tilde{Q}$ satisfies

$$
\begin{aligned}
0 & =\left(\tilde{A}-\gamma^{-1} \tilde{B}_{0} \tilde{C}_{0}\right) \tilde{Q}+\tilde{Q}\left(\tilde{A}-\gamma^{-1} \tilde{B}_{0} \tilde{C}_{0}\right)^{T} \\
& +\tilde{Q}\left[\tilde{B}_{0}^{T} \tilde{P}+Q \tilde{C}_{0}+N \tilde{C}_{0}\left(\tilde{A}-\gamma^{-1} \tilde{B}_{0} \tilde{C}_{0}\right)\right]^{*} \\
& \times\left(\gamma Q-N C_{0} B_{0}-B_{0}^{T} C_{0}^{T} N\right)^{-1} \tilde{B}_{0}^{T} \\
& +\tilde{B}_{0}\left(\gamma Q-N C_{0} B_{0}-B_{0}^{T} C_{0}^{T} N\right)^{-1} \\
& \times\left[\tilde{B}_{0}^{T} \tilde{P}+Q \tilde{C}_{0}+N \tilde{C}_{0}\left(\tilde{A}-\gamma^{-1} \tilde{B}_{0} \tilde{C}_{0}\right)\right] \tilde{Q}+\tilde{D}^{T} \tilde{D}
\end{aligned}
$$


and

$$
0=P_{12}^{T} Q_{12}+P_{2} Q_{2}
$$

$$
\begin{aligned}
0 & =\left(P_{12}^{T} Q_{1}+P_{2} Q_{12}^{T}\right) C^{T}+\left(P_{2} B_{c} D_{2}+P_{12}^{T} D_{1}\right. \\
& \left.+\gamma^{-1} C_{0}^{T} N C_{0} D_{1}\right) D_{2}^{T}
\end{aligned}
$$

$0=B^{T}\left(P_{1} Q_{12}+P_{12} Q_{2}\right)+E_{2}^{T}\left(E_{2} C_{c} Q_{2}+E_{1} Q_{12}\right)$

$$
\begin{aligned}
& +B^{T} C_{0}^{T} N\left(\gamma Q-N C_{0} B_{0}-B_{0}^{T} C_{0}^{T} N\right)^{-1} \\
& \times\left[B_{0}^{T} P_{1}+Q C_{0}+N C_{0}\left(A-\gamma^{-1} B_{0} C_{0}\right)\right] Q_{12} \\
& +B^{T} C_{0}^{T} N\left(\gamma Q-N C_{0} B_{0}-B_{0}^{T} C_{0}^{T} N\right)^{-1} \\
& \times\left(B_{0}^{T} P_{12}+N C_{0} B C_{c}\right) Q_{2}
\end{aligned}
$$

$0=\left(\gamma Q-N C_{0} B_{0}-B_{0}^{T} C_{0}^{T} N\right)^{-1}$

$$
\begin{aligned}
& \times\left[\tilde{B}_{0}^{T} \tilde{P}+Q \tilde{C}_{0}+N \tilde{C}_{0}\left(\tilde{A}-\gamma^{-1} \tilde{B}_{0} \tilde{C}_{0}\right)\right] \tilde{Q} \\
& \times\left[\tilde{B}_{0}^{T} \tilde{P}+Q \tilde{C}_{0}+N \tilde{C}_{0}\left(\tilde{A}-\gamma^{-1} \tilde{B}_{0} \tilde{C}_{0}\right)\right]^{*} \\
& \times\left(\gamma Q-N C_{0} B_{0}-B_{0}^{T} C_{0}^{T} N\right)^{-1} \tilde{B}_{0}^{T} \tilde{C}_{0}^{T} \\
& +\tilde{C}_{0} \tilde{B}_{0}\left(\gamma Q-N C_{0} B_{0}-B_{0}^{T} C_{0}^{T} N\right)^{-1} \\
& \times\left[\tilde{B}_{0}^{T} \tilde{P}+Q \tilde{C}_{0}+N \tilde{C}_{0}\left(\tilde{A}-\gamma^{-1} \tilde{B}_{0} \tilde{C}_{0}\right)\right] \tilde{Q} \\
& \times\left[\tilde{B}_{0}^{T} \tilde{P}+Q \tilde{C}_{0}+N \tilde{C}_{0}\left(\tilde{A}-\gamma^{-1} \tilde{B}_{0} \tilde{C}_{0}\right)\right]^{*} \\
& \times\left(\gamma Q-N C_{0} B_{0}-B_{0}^{T} C_{0}^{T} N\right)^{-1} \\
& +\left(\gamma Q-N C_{0} B_{0}-B_{0}^{T} C_{0}^{T} N\right)^{-1} \\
& \times\left[\tilde{B}_{0}^{T} \tilde{P}+Q \tilde{C}_{0}+N \tilde{C}_{0}\left(\tilde{A}-\gamma^{-1} \tilde{B}_{0} \tilde{C}_{0}\right)\right] \\
& \times \bar{Q}\left(\tilde{A}-\gamma^{-1} \tilde{B}_{0} \tilde{C}_{0}\right)^{T} \tilde{C}_{0}^{T}+\tilde{C}_{0}\left(\tilde{A}-\gamma^{-1} \tilde{B}_{0} \tilde{C}_{0}\right) \tilde{Q} \\
& \times\left[\tilde{B}_{0}^{T} \tilde{P}+Q \tilde{C}_{0}+N \tilde{C}_{0}\left(\tilde{A}-\gamma^{-1} \tilde{B}_{0} \tilde{C}_{0}\right)\right]^{*} \\
& \times\left(\gamma Q-N C_{0} B_{0}-B_{0}^{T} C_{0}^{T} N\right)^{-1}+\gamma^{-1} C_{0} D_{1} D_{1}^{T} C_{0}^{T} \\
& 0=\left(\gamma Q-N C_{0} B_{0}-B_{0}^{T} C_{0}^{T} N\right)^{-1} \\
& \times\left[\tilde{B}_{0}^{T} \tilde{P}+Q \tilde{C}_{0}+N \tilde{C}_{0}\left(\tilde{A}-\gamma^{-1} \tilde{B}_{0} \tilde{C}_{0}\right)\right] \tilde{Q} \tilde{C}_{0}^{T} \\
& +\tilde{C}_{0} \tilde{Q}\left[\tilde{B}_{0}^{T} \tilde{P}+Q \tilde{C}_{0}+N \tilde{C}_{0}\left(\tilde{A}-\gamma^{-1} \tilde{B}_{0} \tilde{C}_{0}\right)\right]^{*} \\
& \times\left(\gamma Q-N C_{0} B_{0}-B_{0}^{T} C_{0}^{T} N\right)^{-1} \\
& -\gamma\left(\gamma Q-N C_{0} B_{0}-B_{0}^{T} C_{0}^{T} N\right)^{-1} \\
& \times\left[\tilde{B}_{0}^{T} \tilde{P}+Q \tilde{C}_{0}+N \tilde{C}_{0}\left(\tilde{A}-\gamma^{-1} \tilde{B}_{0} \tilde{C}_{0}\right)\right] \tilde{Q} \\
& \times\left[\tilde{B}_{0}^{T} \tilde{P}+Q \tilde{C}_{0}+N \tilde{C}_{0}\left(\tilde{A}-\gamma^{-1} \tilde{B}_{0} \tilde{C}_{0}\right)\right]^{*} \\
& \times\left(\gamma Q-N C_{0} B_{0}-B_{0}^{T} C_{0}^{T} N\right)^{-1}
\end{aligned}
$$

Remark 1: The controller gains $A_{c}, B_{c}$, and $C_{c}$, the multiplier matrix $N$, and the scaling matrix $Q$ can be found by means of a quasi-Newton optimization algorithm that uses the partial derivatives of the Lagrangian. These partial derivatives are given by the necessary conditions (10-14), and where $\tilde{P}$ and $\tilde{Q}$ satisfy Eqs. (7) and (9), respectively. Section $V$ includes several numerical examples to illustrate this optimization procedure.
Remark 2: The robust controller procedure presented here allows the controller gains $A_{c}, B_{c}$, and $C_{c}$, the multiplier matrix $N$, and the scaling matrix $Q$ to be found simultaneously. This procedure is, thus, distinct from the $D-K$ iteration procedure of $\mu$ synthesis and the $D, G-K$ iteration procedure of mixed $\mu$ synthesis, where alternate steps are used to find the controller gains and the multiplier and scaling matrices. The idea of computing the gradient of the Lagrangian with respect to the multiplier matrix $N$ presented in Haddad and Berstein $^{3}$ is extended here to include the scaling matrix $Q$.

Remark 3: Robust controller synthesis using a generalized version of the Popov criterion for real, diagonal uncertainty was studied in How et al. ${ }^{8}$ and Haddad et al. ${ }^{9}$ with a multiplier of the form $H+s N$, where $H$ is a diagonal, positive-definite matrix and $N$ is a diagonal, nonnegative-definite matrix. The controller presented in Theorem 2 utilizes the scaled Popov criterion for real, blockdiagonal uncertainty ${ }^{6}$ with the Popov multiplier $I+s N$, where $N$ is a Hermitian, block-diagonal matrix that may be indefinite. In addition, the positive-definite, block-diagonal scaling matrix $Q$ used in Theorem 2 to reduce conservatism does not appear in How et al. ${ }^{8}$ or Haddad et al. ${ }^{9}$

\section{Numerical Examples}

In this section we present several numerical examples to demonstrate the use of Theorem 2 for solving the auxiliary minimization problem. We use the necessary conditions (10-14) from Theorem 2 with the quasi-Newton optimization algorithm uncmnd.f from Kahaner et al. ${ }^{10}$ and Dennis et al. ${ }^{11}$ to compute the controller gains $A_{c}, B_{c}$, and $C_{c}$, the multiplier matrix $N$, and the scaling matrix $Q$ that minimize the auxiliary cost in Eq. (8) subject to the Riccati equation (7) of the scaled Popov criterion.

In Theorem 2, the necessary conditions (10-14) correspond to the gradients of the Lagrangian with respect to the controller parameters $A_{c}, B_{c}$, and $C_{c}$, the multiplier matrix $N$, and the scaling matrix $Q$, respectively, whereas the Lyapunov equation (9) arises from the gradient of the Lagrangian with respect to $\tilde{P}$. These gradients can be computed at each iteration by solving Eqs. (7) and (9) and using Eqs. (10-14). It follows from Proposition 1 of Wang and Bernstein $^{12}$ that if Eqs. (7) and (9) are satisfied, then the gradients of the Lagrangian $\mathcal{L}\left(A_{c}, B_{c}, C_{c}, N, Q\right)$ are equal to the gradients of auxiliary cost $\mathcal{J}\left(A_{c}, B_{c}, C_{c}, N, Q\right)$.

\section{A. Three-Mass System}

Consider the dynamic system in Fig. 1 consisting of three masses and two springs. ${ }^{3}$ The control force acts on the third mass, whereas the disturbance force acts on the first mass. In addition, there is white noise corrupting the measurement $y=x_{1}+x_{2}+w$. Assume that $m_{1}=m_{2}=m_{3}=1, k_{1}=1$, and $k_{2}=k_{2, \text { nom }}+\delta$, where $k_{2, \text { nom }}=1$ and $\delta$ represents the uncertainty in the stiffness of the second spring. The equations of motion are given by

$$
\begin{aligned}
& \dot{x}=\left[\begin{array}{ccccc}
0 & 0 & 0 & 1 & 0 \\
0 & 0 & 0 & 0 & 1 \\
0 & 0 & 0 & 0 & 0 \\
-k_{1} / m_{1} & k_{1} / m_{1} & 0 & 0 & 0 \\
k_{1} / m_{2} & -\left(k_{1}+k_{2}\right) / m_{2} & k_{2} / m_{2} & 0 & 0 \\
0 & k_{2} / m_{3} & -k_{2} / m_{3} & 0 & 0
\end{array}\right. \\
&+ {\left[\begin{array}{cc}
0 & 0 \\
0 & 0 \\
0 & 0 \\
1 / m_{1} & 0 \\
0 & 0 \\
0 & 0
\end{array}\right] w+\left[\begin{array}{c}
0 \\
0 \\
0 \\
0 \\
0 \\
1 / m_{3}
\end{array}\right] u } \\
& z=\left[\begin{array}{llllll}
1 & 1 & 0 & 0 & 0 & 0 \\
0 & 0 & 0 & 0 & 0 & 0
\end{array}\right] x+\left[\begin{array}{l}
0 \\
1
\end{array}\right] u \\
& y=\left[\begin{array}{llllll}
1 & 1 & 0 & 0 & 0 & 0
\end{array}\right] x+\left[\begin{array}{ll}
0 & 1
\end{array}\right] w
\end{aligned}
$$




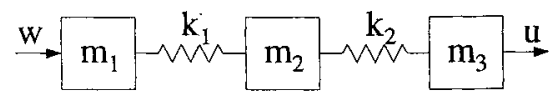

Fig. 1 Three-mass system.

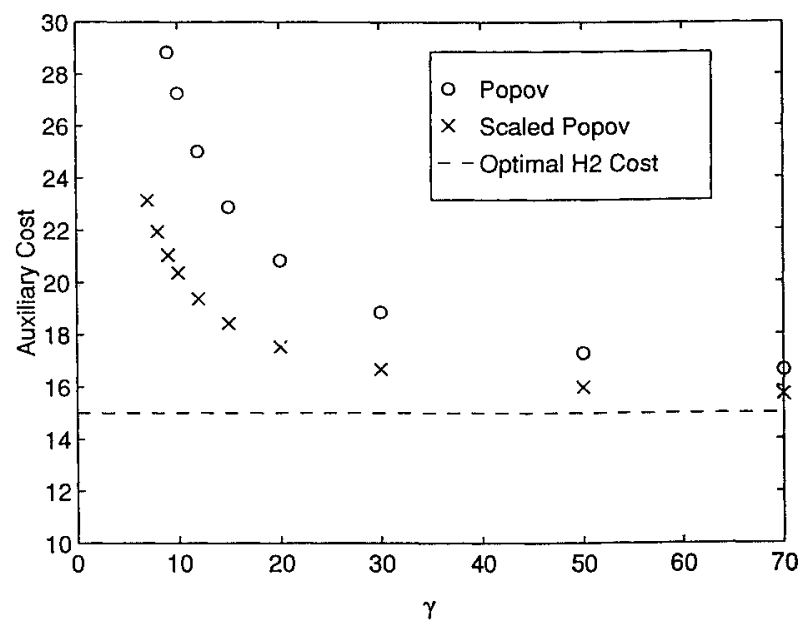

Fig. 2 Auxiliary cost vs $\gamma$ for the three-mass system.

whereas to represent the stiffness uncertainty $\delta$, the matrices $B_{0}$ and $C_{0}$ are given by

$$
B_{0}=\left[\begin{array}{llllll}
0 & 0 & 0 & 0 & -1 & 1
\end{array}\right]^{T}, \quad C_{0}=\left[\begin{array}{llllll}
0 & 1 & -1 & 0 & 0 & 0
\end{array}\right]
$$

The quasi-Newton optimization algorithm was used to compute full-order $\left(n_{c}=6\right)$ controllers that minimize the auxiliary cost $\mathcal{J}\left(\gamma, A_{\mathrm{c}}, B_{c}, C_{c}, N, Q\right)$ for different values of $\gamma$. The linear quadratic Gaussian (LQG) compensator, which corresponds to $\gamma=\infty$, was used to initialize the controller parameters $A_{c}, B_{c}$, and $C_{c}$. To demonstrate the effect of the scaling matrix $Q$ on the conservatism of the controller, we first set $Q$ to be the identity matrix and performed the optimization with respect to the control gains $A_{c}, B_{c}$, and $C_{c}$ and the multiplier matrix $N$. A large initial value of $\gamma$, which corresponds to a small amount of parameter uncertainty, was chosen for the first iteration. The initial value of the multiplier matrix $N$ was computed by using Schur complements to write the Riccati equation (7) as an LMI and finding a feasible matrix $N$ that satisfies it. ${ }^{13}$ This guarantees that the Riccati equation (7) has a positive-definite solution. The gradient optimization was performed until the gradient became small, indicating that a solution near an extremal had been found. The value of $\gamma$ was then reduced, and the previous solution was used to initialize the next iteration.

To improve performance, the auxiliary cost was also optimized with respect to the scaling matrix $Q$. To guarantee that the Riccati equation (7) has a solution, the multiplier and scaling matrices $N$ and $Q$ were initialized by finding feasible matrices $N$ and $Q$ that satisfy the corresponding LMI. The synthesis proceeded as before, by reducing $\gamma$ at each step and using the previous solution to initialize the gains for the next iteration. The auxiliary costs vs the uncertainty bounds $\gamma$ for each controller with and without the scaling matrix $Q$ are shown in Fig. 2. As can be seen, the upper bound for the worst-case $\mathrm{H}_{2}$ norm of the closed-loop system increases as $\gamma$ decreases, that is, as the allowable set of perturbations increases. Furthermore, it can be seen from Fig. 2 that the scaling matrix $Q$ reduces the conservatism of the controllers, since a lower auxiliary cost is obtained for a given $\gamma$.

Finally, the actual $\mathrm{H}_{2}$ performance of the closed-loop system was computed for a range of the uncertain stiffness $k_{2}=1+\delta$ for each of four different controllers, namely, the LQG controller and threescaled Popov controllers with $\gamma=20,9$, and 7. These controllers are given in Appendix B, and the $\mathrm{H}_{2}$ costs of the closed-loop system are shown in Fig. 3. Since the $\mathrm{H}_{2}$ cost is guaranteed to be less than the auxiliary cost for all $k_{2} \in\left[1-\gamma^{-1}, 1+\gamma^{-1}\right]$, a robust performance bound given by the auxiliary cost is guaranteed a priori in that range. As $\gamma$ decreases, the $H_{2}$ norm of the nominal closed-loop system with $k_{2}=k_{2 \text {, nom }}=1$ increases, whereas the $H_{2}$ norm of the perturbed

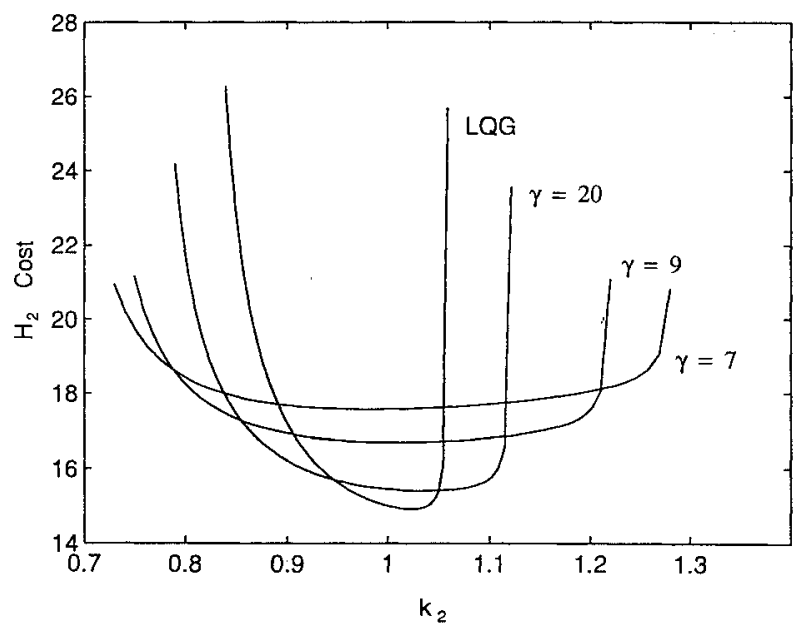

Fig. 3 Dependence of the $\mathrm{H}_{2}$ cost of the closed-loop three-mass system on $k_{2}$.

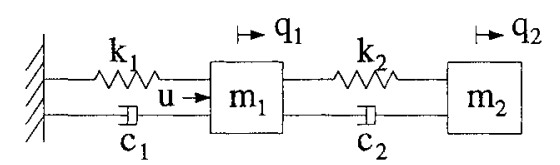

Fig. 4 Two-mass system.

closed-loop system remains close to the nominal value for a range of perturbations $\delta$.

\section{B. Collocated Two-Mass System}

Consider the dynamic system shown in Fig. 4 that represents a flexible structure with uncertain high-frequency dynamics. This example was used in Friedman and Bernstein ${ }^{7}$ to demonstrate the properties of maximum entropy controllers on collocated and noncollocated systems. The equations of motion for this system are

$$
\begin{gathered}
m_{1} \ddot{q}_{1}+c_{1} \dot{q}_{1}-c_{2}\left(\dot{q}_{2}-\dot{q}_{1}\right)+k_{1} q_{1}-k_{2}\left(q_{2}-q_{1}\right)=u \\
m_{2} \ddot{q}_{2}+c_{2}\left(\dot{q}_{2}-\dot{q}_{1}\right)+k_{2}\left(q_{2}-q_{1}\right)=0
\end{gathered}
$$

We first consider the case of a collocated sensor and actuator pair, where the output is given by $y_{\mathrm{col}}=\dot{q}_{1}$. Letting $m_{1}=1, m_{2}=10$, $k_{1}=k_{2}=1$, and $c_{1}=c_{2}=0.01$ and transforming to real normal coordinates yields the plant state space realization

$$
\begin{aligned}
\dot{x} & =\left[\begin{array}{cccc}
-0.0002 & 0.2208 & 0 & 0 \\
-0.2208 & -0.0002 & 0 & 0 \\
0 & 0 & -0.0103 & 1.4320 \\
0 & 0 & -1.4320 & -0.0103
\end{array}\right] x \\
+ & {\left[\begin{array}{r}
-0.1439 \\
0.2168 \\
-0.0426 \\
1.1890
\end{array}\right] u } \\
& y_{\mathrm{col}}=\left[\begin{array}{llll}
-0.0545 & 0.0819 & -0.0352 & 0.8181] x
\end{array}\right.
\end{aligned}
$$

The matrices $D_{1}, D_{2}, E_{1}$, and $E_{2}$ are chosen to be

$$
\begin{gathered}
D_{1}=\left[\begin{array}{ll}
0 & 0 \\
1 & 0 \\
0 & 0 \\
0 & 0
\end{array}\right], \quad D_{2}=\left[\begin{array}{ll}
0 & 1
\end{array}\right], \quad E_{1}=\left[\begin{array}{llll}
1 & 0 & 0 & 0 \\
0 & 0 & 0 & 0
\end{array}\right] \\
E_{2}=\left[\begin{array}{l}
0 \\
1
\end{array}\right]
\end{gathered}
$$



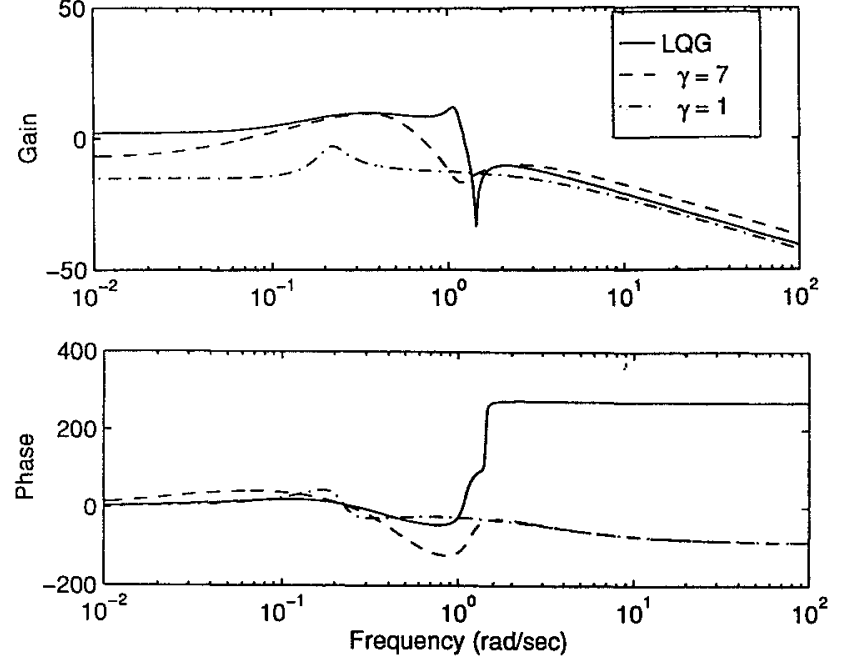

Fig. 5 Frequency responses of scaled Popov controllers for collocated two-mass system.

so that the LQG compensator places a notch at the second modal frequency. Uncertainty in the damped natural frequency of the second mode $\omega_{d 2}=1.432$ is modeled by choosing

$$
B_{0}=\left[\begin{array}{rr}
0 & 0 \\
0 & 0 \\
0 & 1 \\
-1 & 0
\end{array}\right], \quad C_{0}=\left[\begin{array}{llll}
0 & 0 & 1 & 0 \\
0 & 0 & 0 & 1
\end{array}\right]
$$

Hence, the perturbed dynamics matrix is $A+B_{0} \Delta C_{0}$, where $\Delta=$ $\operatorname{diag}(\delta, \delta)$ and $\delta$ represents uncertainty in $\omega_{d 2}$.

The quasi-Newton optimization algorithm was used as before to compute full-order controllers $\left(n_{c}=4\right)$ that minimize the auxiliary cost for several values of $\gamma$. The frequency responses of the LQG controller and the two scaled Popov controllers with $\gamma=7$ and $\gamma=1$ are shown in Fig. 5. The LQG controller is unstable and achieves closed-loop stability and nominal performance by placing a notch at the nominal damped natural frequency $\omega_{d 2}$ of the uncertain second mode. Hence, closed-loop performance degrades considerably when the damped natural frequency of the second mode is perturbed. The scaled Popov controllers with $\gamma=7$ and $\gamma=1$ are asymptotically stable, but the controller with $\gamma=7$ has only a shallow notch near the damped natural frequency of the second mode, whereas the controller with $\gamma=1$ has no notch near that frequency. Hence, these controllers sacrifice nominal performance for improved robust performance over a larger range of the uncertain damped natural frequency. As $\gamma$ decreases, the controllers guarantee robust performance over a larger range of $\delta$. Note that the controller obtained with $\gamma=1$ is positive real. Since the plant is a model of a flexible structure with a collocated sensor and actuator pair, it is also positive real and, thus, the closed-loop system is asymptotically stable for all values of the uncertain damped natural frequency.

The actual $\mathrm{H}_{2}$ cost was computed for a range of values of the damped natural frequency of the second mode for the LQG controller and for the three scaled Popov controllers corresponding to $\gamma=15,7$, and 2. These controllers are given in Appendix C, and the cost dependence is shown in Fig. 6. As $\gamma$ decreases, the $H_{2}$ cost of the nominal closed-loop system increases whereas the $\mathrm{H}_{2}$ cost of the perturbed closed-loop system remains near the nominal value for a larger range of perturbations. The LQG controller stabilizes the closed-loop system for only small perturbations in the damped natural frequency of the second mode, whereas the scaled Popov controllers stabilize the closed-loop system and provide performance close to the optimal level even for large perturbations. Hence, robust performance over a large range of the uncertain parameter is achieved for only a small increase in the $\mathrm{H}_{2}$ cost above the optimal.

It is interesting to compare the scaled Popov controllers with the maximum entropy controllers given in Friedman and Bernstein. ${ }^{7}$ As

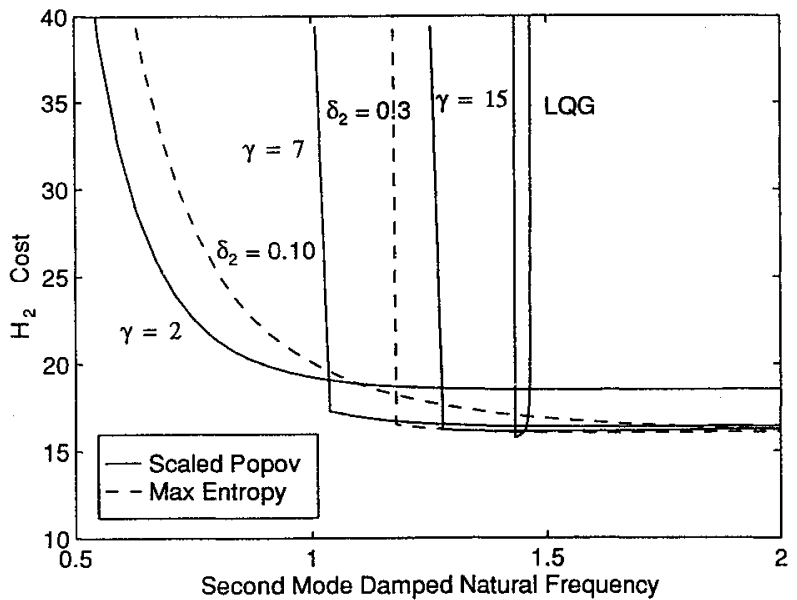

Fig. 6 Dependence of $\mathrm{H}_{2}$ cost on the damped natural frequency of the second mode for the collocated two-mass system.

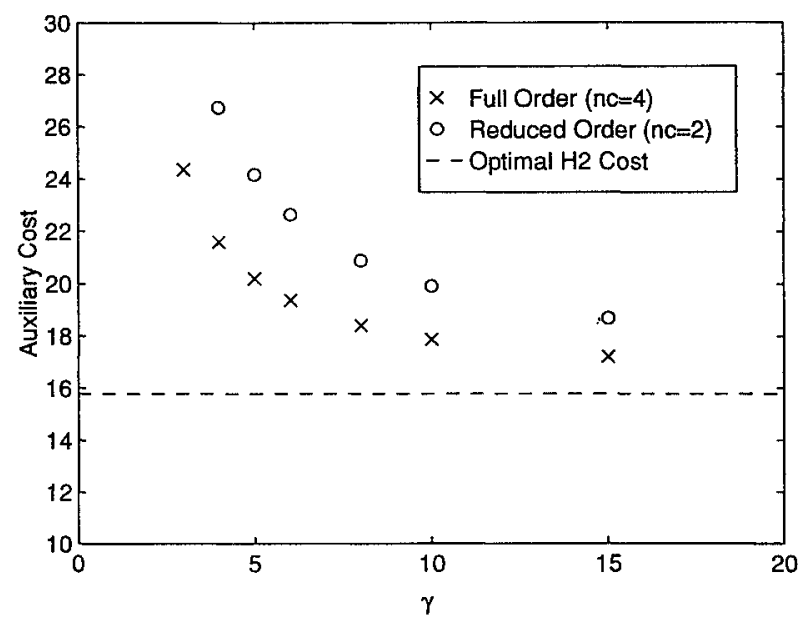

Fig. 7 Auxiliary cost vs $\gamma$ for scaled Popov controllers for the collocated two-mass system.

discussed in Friedman and Bernstein ${ }^{7}$ and Bernstein and Hyland, ${ }^{14}$ this technique also yields robust controllers for flexible structures with uncertain damped natural frequencies. Furthermore, as shown in Friedman and Bernstein, ${ }^{7}$ as the uncertainty level is increased, the maximum entropy controllers tend to become positive real. Until now, the maximum entropy method was the only technique known to yield positive real controllers for positive real plants as a direct consequence of uncertainty. As this example shows, however, scaled Popov synthesis also yields positive real controllers for positive real plants. The gains of the maximum entropy controllers in Friedman and Bernstein ${ }^{7}$ for $\delta_{2}=0.3$ and 10 were obtained from the authors and are given in Appendix C. The actual $H_{2}$ costs for the maximum entropy controllers are shown in Fig. 6 with the actual $H_{2}$ costs for the scaled Popov controllers. Although the parameter $\delta_{2}$ is not a bound on the uncertainty $\delta$, it can be seen from Fig. 6 that increasing $\delta_{2}$ in maximum entropy controller synthesis has the same effect as decreasing $\gamma$ in scaled Popov controller synthesis.

The scaled Popov synthesis technique was also used to compute reduced-order controllers $\left(n_{c}=2\right)$ for the flexible structure with the collocated sensor and actuator pair. A reduced-order controller that stabilizes the two-mass system is required to initialize the gradient search. Although the balanced truncation of the LQG controller did not stabilize the two-mass system, the balanced truncation of the full-order scaled Popov controller with $\gamma=10$ was found to be stabilizing and was, thus, used to initialize the reduced-order controller gains. The numerical optimization proceeded as before, with decreasing $\gamma$ and with each solution used to initialize the next iteration. The auxiliary costs vs the robustness bounds $\gamma$ for each reduced-order controller are shown in Fig. 7 with the auxiliary costs vs the robustness bounds $\gamma$ for each full-order 

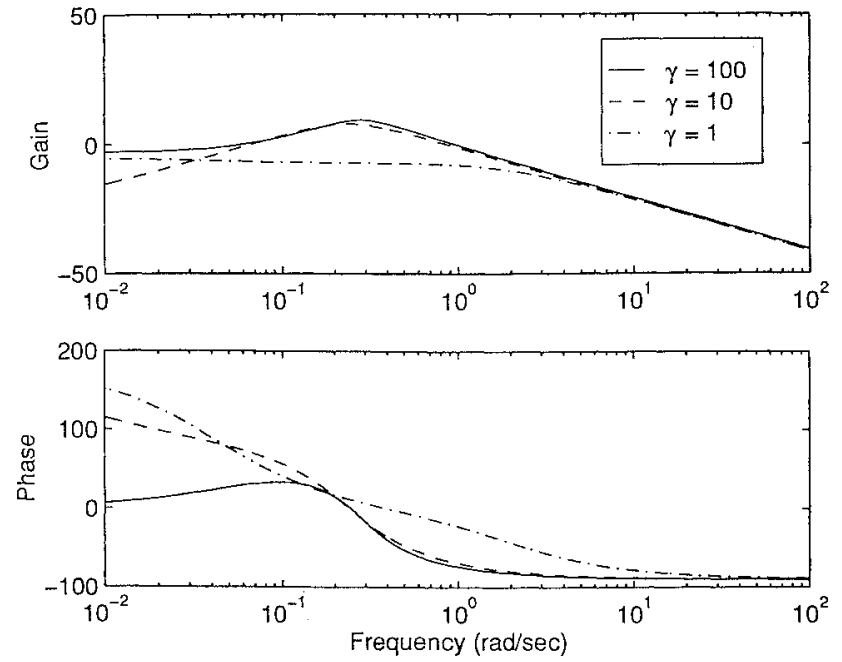

Fig. 8 Frequency responses of reduced-order scaled Popov controllers for the collocated two-mass system.
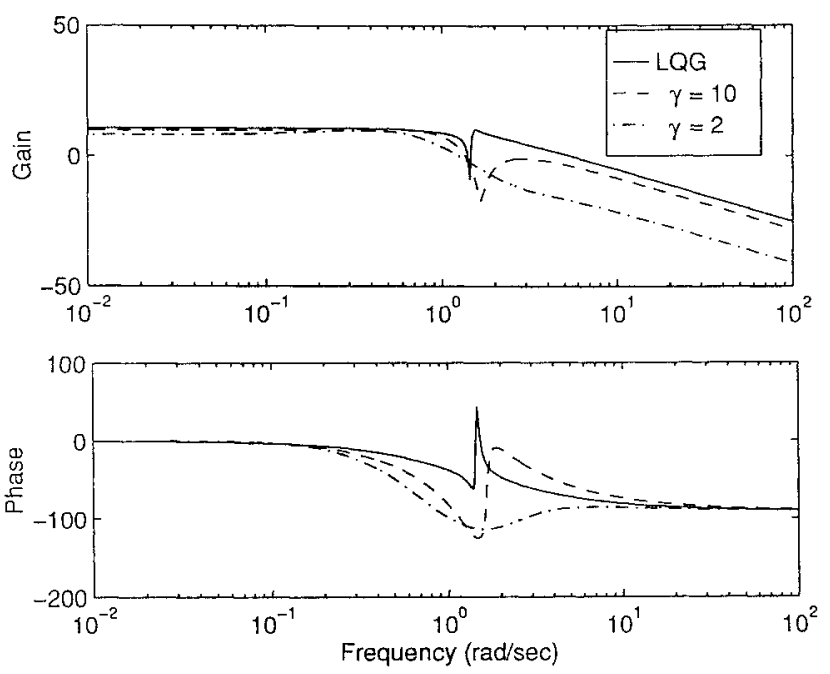

Fig. 9 Frequency responses of scaled Popov controllers for noncollocated two-mass system.

controller. Clearly, the costs obtained for the full-order controllers are lower than the costs for the reduced-order controller for the corresponding values of $\gamma$. The frequency responses of the reducedorder scaled Popov controllers with $\gamma=100,10$, and 1 are shown in Fig. 8.

\section{Noncollocated Two-Mass System}

Next we consider the two-mass system of Fig. 4 with a noncollocated sensor and actuator pair by choosing $y_{\text {noncol }}=\dot{q}_{2}$, so that

$$
y_{\text {noncol }}=\left[\begin{array}{llll}
-0.1063 & 0.1597 & 0.0018 & -0.0419
\end{array}\right] x
$$

We increase the matrix $E_{1}$ by a factor of 10 to enhance the notching characteristics of the LQG controller. ${ }^{7}$

The scaled Popov controller synthesis technique was used as before to compute full-order controllers $\left(n_{c}=4\right)$ that solve the auxiliary minimization problem for this uncertain plant for a range of $\gamma$. The frequency responses of the LQG controller and the scaled Popov controllers with $\gamma=10$ and $\gamma=2$ were computed and shown in Fig. 9. Since the plant is not positive real, robust performance cannot be achieved by positive real controllers, as in the collocated case. Instead, as seen in Fig. 9, the controllers widen and deepen the notch at the nominal frequency of the uncertain mode.

The actual $\mathrm{H}_{2}$ cost was computed for a range of the damped natural frequency for the LQG controller and for three scaled Popov controllers corresponding to $\gamma=15,4$, and 2 . These controllers are

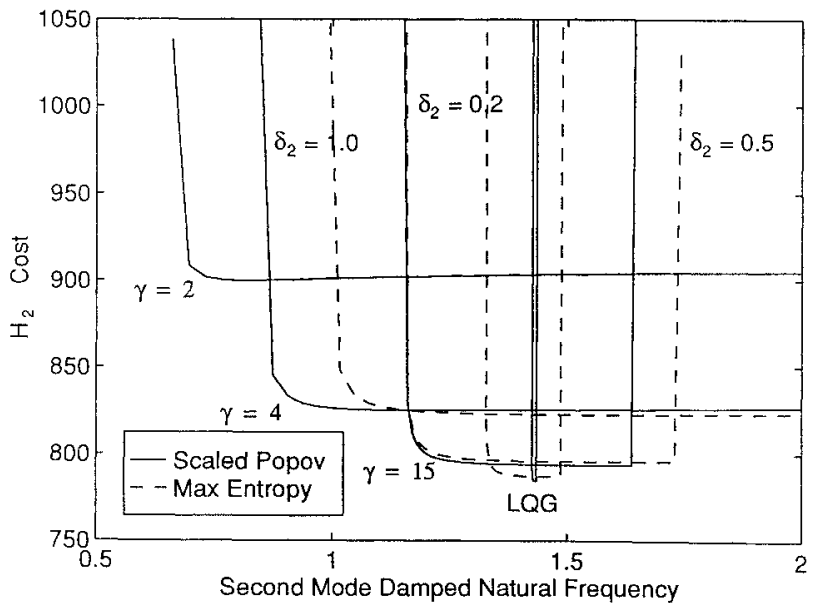

Fig. 10 Dependence of $\mathrm{H}_{2}$ cost on $\delta$ for controllers for noncollocated two-mass system.

given in Appendix D and the cost dependence is shown in Fig. 10. In the noncollocated case, the LQG controller stabilizes the plant only for a narrow range of the uncertain parameter. In fact, the LQG controller for the noncollocated plant stabilizes the plant for a smaller range of the uncertain parameter than the unstable LQG controller for the collocated plant. The maximum entropy controllers from Friedman and Bernstein ${ }^{7}$ with $\delta_{2}=0.2,0.5$, and 1.0 are given in Appendix D. The actual $H_{2}$ cost was computed for a range of the damped natural frequency for these controllers and also appears in Fig. 10. As in the collocated case, the increasing $\delta_{2}$ with the maximum entropy controllers has the same effect as decreasing $\gamma$ with the scaled Popov controllers.

\section{Conclusions}

A robust controller synthesis procedure for plants with linear, time-invariant, norm-bounded, block-structured uncertainty and a numerical algorithm for its solution were presented. The worst-case $\mathrm{H}_{2}$ norm over the set of allowable real perturbations was written in terms of the observability Gramian of the uncertain closed-loop system. Then, using the scaled Popov criterion, an upper bound for the worst-case $\mathrm{H}_{2}$ norm was derived. The gradients of the Lagrangian with respect to the control gains, the matrix multiplier, and the scaling matrix were then used with a quasi-Newton optimization algorithm to find controllers that minimize the upper bound for the worst-case $\mathrm{H}_{2}$ norm. The quasi-Newton optimization algorithm converged at each iteration when the gradient vector was sufficiently small. The number of optimization steps varied with problem size and the amount that $\gamma$ is decreased and was generally between 50 and 300 steps. Hence, the controller could be efficiently computed at each iteration.

The numerical examples demonstrated the robust performance achieved using these controllers. For positive real plants with sufficiently large real parameter uncertainty, the synthesis procedure was shown to yield positive real controllers, thus guaranteeing closedloop stability for all perturbations such that the plant remains positive real. Until now, maximum entropy controller synthesis was the only procedure that was known to yield positive real controllers for positive real plants as a direct result of model uncertainty.

There are several possible directions for future work in this area. First is a refinement of the scaled Popov criterion to reduce conservatism of the robustness bound. This would entail more general multipliers and scalings than the ones presented here. Second, the actual $\mathrm{H}_{2}$ cost, rather than the auxiliary cost, could be minimized. Either of these directions would lead to a controller synthesis procedure with improved robust performance for real parameter uncertainty. Finally, motivated by the similarity of scaled Popov controllers and the maximum entropy controllers for positive real plants such as flexible structures, the framework used here to derive scaled Popov controllers could be used to obtain a rigorous framework for maximum entropy controller synthesis. 


\section{Appendix A: Proofs of Theorems 1 and 2}

\section{Proof of Theorem 1}

Since $\gamma Q-N C_{0} B_{0}-B_{0}^{T} C_{0}^{T} N>0$ and

$$
\begin{aligned}
0 & >\left(A-\gamma^{-1} B_{0} C_{0}\right)^{T} P+P\left(A-\gamma^{-1} B_{0} C_{0}\right) \\
& +\left[B_{0}^{T} P+Q C_{0}+N C_{0}\left(A-\gamma^{-1} B_{0} C_{0}\right)\right]^{*} \\
& \times\left(\gamma Q-N C_{0} B_{0}-B_{0}^{T} C_{0}^{T} N\right)^{-1} \\
& \times\left[B_{0}^{T} P+Q C_{0}+N C_{0}\left(A-\gamma^{-1} B_{0} C_{0}\right)\right]=-R
\end{aligned}
$$

it follows using Schur complements that

$$
\left[\begin{array}{c}
\left(A-\gamma^{-1} B_{0} C_{0}\right)^{T} P+P\left(A-\gamma^{-1} B_{0} C_{0}\right) \\
B_{0}^{T} P+Q C_{0}+N C_{0}\left(A-\gamma^{-1} B_{0} C_{0}\right)
\end{array}\right.
$$

Asymptotic stability of the feedback interconnection of $G(s)=$ $C_{0}(s I-A)^{-1} B_{0}$ and $\Delta$ for all $\Delta \in \Delta_{\gamma}$ follows from Eq. (A1) and Theorem 2 of Sparks and Berstein. ${ }^{6}$ Hence, $A+B_{0} \Delta C_{0}$ is Hurwitz for all $\Delta \in \Delta_{\gamma}$.

Next, rewriting Eq. (5) by adding and subtracting $\left(B_{0} \Delta C_{0}\right)^{T} P+$ $P B_{0} \triangle C_{0}$ yields

$$
\begin{aligned}
0 & =\left(A+B_{0} \Delta C_{0}\right)^{T} P+P\left(A+B_{0} \Delta C_{0}\right) \\
& +\left[B_{0}^{T} P+Q C_{0}+N C_{0}\left(A-\gamma^{-1} B_{0} C_{0}\right)\right]^{*} \\
& \times\left(\gamma Q-N C_{0} B_{0}-B_{0}^{T} C_{0}^{T} N\right)^{-1} \\
& \times\left[B_{0}^{T} P+Q C_{0}+N C_{0}\left(A-\gamma^{-1} B_{0} C_{0}\right)\right] \\
& +R-\left[B_{0}\left(\Delta+\gamma^{-1} I\right) C_{0}\right]^{T} P-P B_{0}\left(\Delta+\gamma^{-1} I\right) C_{0}
\end{aligned}
$$

Furthermore, adding and subtracting $\left(A+B_{0} \Delta C_{0}\right)^{T} C_{0}^{T}(\Delta+$ $\left.\gamma^{-1} I\right) N C_{0}+C_{0}^{T}\left(\Delta+\gamma^{-1} I\right) N C_{0}\left(A+B_{0} \Delta C_{0}\right)$ yields

$$
\begin{aligned}
0 & =\left(A+B_{0} \Delta C_{0}\right)^{T}\left[P+C_{0}^{T}\left(\Delta+\gamma^{-1} I\right) N C_{0}\right] \\
& +\left[P+C_{0}^{T}\left(\Delta+\gamma^{-1} I\right) N C_{0}\right]\left(A+B_{0} \Delta C_{0}\right)+R+\Lambda
\end{aligned}
$$

where

$$
\begin{aligned}
\Lambda & =\left[B_{0}^{T} P+Q C_{0}+N C_{0}\left(A-\gamma^{-1} B_{0} C_{0}\right)\right]^{*} \\
& \times\left(\gamma Q-N C_{0} B_{0}-B_{0}^{T} C_{0}^{T} N\right)^{-1} \\
& \times\left[B_{0}^{T} P+Q C_{0}+N C_{0}\left(A-\gamma^{-1} B_{0} C_{0}\right)\right] \\
& -\left[B_{0}\left(\Delta+\gamma^{-1} I\right) C_{0}\right]^{T} P-P B_{0}\left(\Delta+\gamma^{-1} I\right) C_{0} \\
& -\left(A+B_{0} \Delta C_{0}\right)^{T} C_{0}^{T}\left(\Delta+\gamma^{-1} I\right) N C_{0} \\
& -C_{0}^{T}\left(\Delta+\gamma^{-1} I\right) N C_{0}\left(A+B_{0} \Delta C_{0}\right)
\end{aligned}
$$

Adding and subtracting $\gamma^{-1} C_{0}^{T}\left(\Delta+\gamma^{-1}\right) N C_{0} B_{0} C_{0}+\gamma^{-1} C_{0}^{T}$ $B_{0}^{T} C_{0}^{T} N\left(\Delta+\gamma^{-1}\right) C_{0}$ to and from $\Lambda$ and rearranging yields

$$
\begin{aligned}
\Lambda & =\left[B_{0}^{T} P+Q C_{0}+N C_{0}\left(A-\gamma^{-1} B_{0} C_{0}\right)\right]^{*} \\
& \times\left(\gamma Q-N C_{0} B_{0}-B_{0}^{T} C_{0}^{T} N\right)^{-1} \\
& \times\left[B_{0}^{T} P+Q C_{0}+N C_{0}\left(A-\gamma^{-1} B_{0} C_{0}\right)\right] \\
& -\left[B_{0}\left(\Delta+\gamma^{-1} I\right) C_{0}\right]^{T} P-P B_{0}\left(\Delta+\gamma^{-1} I\right) C_{0} \\
& -C_{0}^{T}\left(\Delta+\gamma^{-1} I\right) N C_{0}\left(A-\gamma^{-1} B_{0} C_{0}\right) \\
& -\left(A-\gamma^{-1} B_{0} C_{0}\right) C_{0}^{T} N\left(\Delta+\gamma^{-1} I\right) C_{0} \\
& -C_{0}^{T}\left(\Delta+\gamma^{-1} I\right) N C_{0} B_{0}\left(\Delta+\gamma^{-1} I\right) C_{0} \\
& -C_{0}^{T}\left(\Delta+\gamma^{-1} I\right) B_{0}^{T} C_{0}^{T} N\left(\Delta+\gamma^{-1} I\right) C_{0}
\end{aligned}
$$

whereas adding and subtracting $2 C_{0}^{T} Q\left(\Delta+\gamma^{-1}\right) C_{0}+\gamma C_{0}^{T}(\Delta+$ $\left.\gamma^{-1}\right) Q\left(\Delta+\gamma^{-1}\right) C_{0}$ to and from $\Lambda$ yields

$$
\begin{aligned}
\Lambda & =\left[B_{0}^{T} P+Q C_{0}+N C_{0}\left(A-\gamma^{-1} B_{0} C_{0}\right)\right]^{*} \\
& \times\left(\gamma Q-N C_{0} B_{0}-B_{0}^{T} C_{0}^{T} N\right)^{-1} \\
& \times\left[B_{0}^{T} P+Q C_{0}+N C_{0}\left(A-\gamma^{-1} B_{0} C_{0}\right)\right] \\
& -C_{0}^{T}\left(\Delta+\gamma^{-1} I\right)\left[B_{0}^{T} P+Q C_{0}+N C_{0}\left(A-\gamma^{-1} B_{0} C_{0}\right)\right] \\
& -\left[B_{0}^{T} P+Q C_{0}+N C_{0}\left(A-\gamma^{-1} B_{0} C_{0}\right)\right]^{*}\left(\Delta+\gamma^{-1} I\right) C_{0}
\end{aligned}
$$

$\left.\begin{array}{c}P B_{0}+C_{0}^{T} Q+\left(A-\gamma^{-1} B_{0} C_{0}\right)^{T} C_{0}^{T} N \\ N C_{0} B_{0}+B_{0}^{T} C_{0}^{T} N-\gamma Q\end{array}\right]<0$

$$
\begin{aligned}
& +C_{0}^{T}\left(\Delta+\gamma^{-1} I\right)\left(\gamma Q-N C_{0} B_{0}-B_{0}^{T} C_{0}^{T} N\right)\left(\Delta+\gamma^{-1} I\right) C_{0} \\
& +2 C_{0}^{T} Q^{\frac{1}{2}}\left(\Delta+\gamma^{-1} I\right) Q^{\frac{1}{2}} C_{0} \\
& -\gamma C_{0}^{T} Q^{\frac{1}{2}}\left(\Delta+\gamma^{-1} I\right)^{2} Q^{\frac{1}{2}} C_{0} \\
& =\left[B_{0}^{T} P+Q C_{0}+N C_{0}\left(A-\gamma^{-1} B_{0} C_{0}\right)\right. \\
& \left.-\left(\gamma Q-N C_{0} B_{0}-B_{0}^{T} C_{0}^{T} N\right)\left(\Delta+\gamma^{-1} I\right) C_{0}\right]^{*} \\
& \times\left(\gamma Q-N C_{0} B_{0}-B_{0}^{T} C_{0}^{T} N\right)^{-1}\left[B_{0}^{T} P+Q C_{0}\right. \\
& +N C_{0}\left(A-\gamma^{-1} B_{0} C_{0}\right)-\left(\gamma Q-N C_{0} B_{0}-B_{0}^{T} C_{0}^{T} N\right) \\
& \left.\times\left(\Delta+\gamma^{-1} I\right) C_{0}\right]+2 C_{0}^{T} Q^{\frac{1}{2}}\left[\left(\Delta+\gamma^{-1} I\right)\right. \\
& \left.-\frac{1}{2} \gamma\left(\Delta+\gamma^{-1} I\right)^{2}\right] Q^{\frac{1}{2}} C_{0}
\end{aligned}
$$

Next, note that for all $\Delta \in \Delta_{\gamma}, \sigma_{\max }(\Delta) \leq \gamma^{-1}$ so that $-\gamma^{-1} I \leq$ $\Delta \leq \gamma^{-1} I$ and $0 \leq \Delta+\gamma^{-1} I \leq 2 \gamma^{-1} I$. Thus, for all $\Delta \in \Delta_{\gamma}$, $0 \leq \frac{1}{2} \gamma\left(\Delta+\gamma^{-1} I\right) \leq I$, so that $0 \leq\left(\Delta+\gamma^{-1} I\right)-\frac{1}{2} \gamma\left(\Delta+\gamma^{-1} I\right)^{2}$. Hence, since $\gamma Q-N C_{0} B_{0}-B_{0}^{T} C_{0}^{T} N>0$, it follows that $\Lambda \geq 0$. Now, subtracting Eq. (4) from Eq. (A2) yields

$$
\begin{aligned}
0 & =\left(A+B_{0} \Delta C_{0}\right)^{T}\left[P+C_{0}^{T}\left(\Delta+\gamma^{-1} I\right) N C_{0}-P_{\Delta}\right] \\
& +\left[P+C_{0}^{T}\left(\Delta+\gamma^{-1} I\right) N C_{0}-P_{\Delta}\right]\left(A+B_{0} \Delta C_{0}\right) \\
& +\Lambda+R-E^{T} E
\end{aligned}
$$

Since $A+B_{0} \Delta C_{0}$ is Hurwitz for all $\Delta \in \Delta_{\gamma}, R \geq E^{T} E$, and $\Lambda \geq 0$, it follows that $P+C_{0}^{T}\left(\Delta+\gamma^{-1} I\right) N C_{0}-P_{\Delta} \geq 0$ for all $\Delta \in \Delta_{\gamma}$, so that $J(\gamma) \leq \operatorname{tr}\left[P+C_{0}^{T}\left(\Delta+\gamma^{-1} I\right) N C_{0}\right] D D^{T}$ for all $\Delta \in \Delta_{\gamma}$. Finally, since $\Delta+\gamma^{-1} I \leq 2 \gamma^{-1} I$ for all $\Delta \in \Delta_{\gamma}$, it follows that $J(\gamma) \leq \operatorname{tr}\left(P+2 \gamma^{-1} C_{0}^{T} N C_{0}\right) D D^{T}$, which proves Eq. (6).

\section{Proof of Theorem 2}

Forming the Lagrangian by appending the Riccati constraint (7) with Lagrange multiplier $\tilde{Q}$ to the auxiliary cost (8) yields

$$
\begin{aligned}
\mathcal{L} & \left(A_{c}, B_{c}, C_{c}, N, Q\right)=\operatorname{tr}\left(\tilde{P}+2 \gamma^{-1} \tilde{C}_{0}^{T} N \tilde{C}_{0}\right) \tilde{D} \tilde{D}^{T} \\
& +\operatorname{tr} \tilde{Q}\left\{\left(\tilde{A}-\gamma^{-1} \tilde{B}_{0} \tilde{C}_{0}\right)^{T} \tilde{P}+\tilde{P}\left(\tilde{A}-\gamma^{-1} \tilde{B}_{0} \tilde{C}_{0}\right)\right. \\
& +\tilde{E}^{T} \tilde{E}+\left[\tilde{B}_{0}^{T} \tilde{P}+Q \tilde{C}_{0}+N \tilde{C}_{0}\left(\tilde{A}-\gamma^{-1} \tilde{B}_{0} \tilde{C}_{0}\right)\right]^{*} \\
& \times\left(\gamma Q-N \tilde{C}_{0} \tilde{B}_{0}-\tilde{B}_{0}^{T} \tilde{C}_{0}^{T} N\right)^{-1} \\
\times & {\left.\left[\tilde{B}_{0}^{T} \tilde{P}+Q \tilde{C}_{0}+N \tilde{C}_{0}\left(\tilde{A}-\gamma^{-1} \tilde{B}_{0} \tilde{C}_{0}\right)\right]\right\} }
\end{aligned}
$$

Differentiating the Lagrangian with respect to $A_{c}, B_{c}, C_{c}, N$, and $Q$ and setting the derivatives to zero yields the necessary conditions (10-14), whereas differentiating the Lagrangian with respect to $\tilde{P}$ yields the Lyapunov equation (9). 


\section{Appendix B: Controllers for Three-Mass System}

Linear quadratic Gaussian:

$\left[\begin{array}{rrrrrr|r}-0.9305 & -0.9305 & 0.0000 & 1.0000 & 0.0000 & 0.0000 & 0.9305 \\ -0.5829 & -0.5829 & 0.0000 & 0.0000 & 1.0000 & 0.0000 & 0.5829 \\ -0.1517 & -0.1517 & 0.0000 & 0.0000 & 0.0000 & 1.0000 & 0.1517 \\ -1.4820 & 0.5180 & 0.0000 & 0.0000 & 0.0000 & 0.0000 & 0.4820 \\ 0.3368 & -2.6632 & 1.0000 & 0.0000 & 0.0000 & 0.0000 & 0.6632 \\ -0.1744 & 0.7588 & -2.2940 & -1.8337 & -1.5650 & -1.6087 & -0.1452 \\ \hline-0.3196 & -0.3864 & -1.2940 & -1.8337 & -1.5650 & -1.6087 & 0.0000\end{array}\right]$

Scaled Popov with $\gamma=20$ :

$\left[\begin{array}{rrrrrr|r}-0.9613 & -0.9570 & -0.0039 & 0.9961 & -0.0166 & 0.0035 & 0.9691 \\ -0.5942 & -0.6179 & 0.0134 & 0.0107 & 0.9869 & -0.0013 & 0.6546 \\ -0.1862 & -0.1558 & -0.0487 & -0.0174 & -0.0234 & 1.0074 & 0.1423 \\ -1.5180 & 0.5231 & -0.0101 & -0.0328 & -0.0541 & 0.0071 & 0.4955 \\ 0.2924 & -2.6634 & 0.9908 & -0.0217 & -0.0649 & 0.0215 & 0.6674 \\ -0.1995 & 0.7648 & -2.3033 & -1.8521 & -1.5808 & -1.6438 & -0.1464 \\ \hline-0.3156 & -0.3912 & -1.2942 & -1.8515 & -1.5435 & -1.6616 & 0.0000\end{array}\right]$

Scaled Popov with $\gamma=9$ :

$\left[\begin{array}{rrrrrr|r}-1.0043 & -0.9911 & -0.0095 & 0.9906 & -0.0442 & 0.0054 & 1.0181 \\ -0.6129 & -0.6676 & 0.0360 & 0.0240 & 0.9658 & -0.0039 & 0.7447 \\ -0.2247 & -0.1577 & -0.1160 & -0.0439 & -0.0446 & 1.0089 & 0.1251 \\ -1.5610 & 0.5323 & -0.0246 & -0.0787 & -0.1150 & 0.0093 & 0.5028 \\ 0.2364 & -2.6639 & 0.9767 & -0.0527 & -0.1464 & 0.0485 & 0.6628 \\ -0.2245 & 0.7725 & -2.3156 & -1.8757 & -1.5906 & -1.6993 & -0.1500 \\ \hline-0.3086 & -0.3977 & -1.2895 & -1.8694 & -1.5216 & -1.7263 & 0.0000\end{array}\right]$

Scaled Popov with $\gamma=7$ :

$$
\left[\begin{array}{rrrrrr|r}
-1.0285 & -1.0102 & -0.0125 & 0.9873 & -0.0617 & 0.0062 & 1.0445 \\
-0.6254 & -0.6952 & 0.0494 & 0.0306 & 0.9530 & -0.0050 & 0.7928 \\
-0.2431 & -0.1578 & -0.1534 & -0.0593 & -0.0534 & 1.0063 & 0.1144 \\
-1.5828 & 0.5383 & -0.0332 & -0.1041 & -0.1461 & 0.0082 & 0.5029 \\
0.2067 & -2.6642 & 0.9682 & -0.0709 & -0.1890 & 0.0621 & 0.6565 \\
-0.2354 & 0.7769 & -2.3219 & -1.8879 & -1.5928 & -1.7323 & -0.1527 \\
\hline-0.3042 & -0.4011 & -1.2853 & -1.8778 & -1.5110 & -1.7605 & 0.0000
\end{array}\right]
$$

\section{Appendix C: Controllers for Collocated} Two-Mass System

Linear quadratic Gaussian:

$$
\left[\begin{array}{rrrr|r}
-0.0672 & 0.4099 & -0.0213 & 0.4953 & -0.6054 \\
-0.1266 & -0.2751 & 0.0278 & -0.6465 & 0.7902 \\
-0.0101 & 0.0413 & -0.0103 & 1.4322 & -0.0000 \\
0.2808 & -1.1530 & -1.4322 & -0.0103 & -0.0000 \\
\hline 0.2361 & -0.9695 & -0.0000 & -0.0000 & 0.0000
\end{array}\right]
$$

Scaled Popov with $\gamma=15$ :

$\left[\begin{array}{rrrr|r}-0.3455 & 0.5062 & 0.0811 & 0.6705 & -0.1048 \\ -0.0065 & -0.9223 & -0.4018 & -0.3755 & 1.2492 \\ -0.0729 & -0.0702 & -0.2974 & 1.0072 & 0.0536 \\ 0.0663 & -1.0102 & -0.9249 & -0.3736 & 0.0336 \\ \hline 0.9461 & -0.8372 & 0.2545 & -0.2629 & 0.0000\end{array}\right]$

Scaled Popov with $\gamma=7$ :

$\left[\begin{array}{rrrr|r}-1.1079 & 0.1093 & 0.0925 & -0.1214 & -0.0777 \\ -1.9348 & -2.5865 & -0.1292 & -0.8626 & 2.4788 \\ -0.8748 & -1.3756 & 0.6558 & 0.1956 & 0.9374 \\ 2.9296 & 0.8243 & -0.9960 & -0.1035 & -0.4055 \\ \hline 2.5086 & -0.7869 & 0.5753 & -0.5182 & 0.0000\end{array}\right]$

Scaled Popov with $\gamma=2$ :

$\left[\begin{array}{rrrr|r}-0.9997 & -0.2945 & -0.0132 & -0.1500 & 0.2396 \\ -1.6555 & -3.8219 & -0.7282 & -0.7672 & 2.5397 \\ -0.7780 & -1.5166 & 0.5321 & 0.1679 & 0.7929 \\ 3.0180 & 0.3567 & -1.1395 & -0.2423 & -0.0796 \\ \hline 2.4895 & -0.9438 & 0.4749 & -0.3643 & 0.0000\end{array}\right]$


Maximum entropy with $\delta_{2}=0.3$ :

$\left[\begin{array}{rrrr|r}-0.1892 & 0.5358 & 0.0188 & 0.5838 & -0.5061 \\ -0.0082 & -0.6511 & -0.2465 & -0.6252 & 0.9351 \\ -0.0775 & 0.0623 & -0.3564 & 1.3261 & 0.0458 \\ 0.2745 & -1.2288 & -1.3404 & -0.4076 & 0.0324 \\ \hline 0.3819 & -0.9673 & -0.0621 & -0.3439 & 0.0000\end{array}\right]$

Maximum entropy with $\delta_{2}=10$ :

$\left[\begin{array}{rrrr|r}-4.7356 & 4.4517 & -5.5583 & 6.2827 & -0.4490 \\ 3.8619 & -13.1740 & -5.5323 & -11.9357 & 1.9659 \\ -5.8266 & -4.8516 & -18.6345 & 1.3889 & 1.3625 \\ 6.5993 & -11.8794 & 1.3062 & -13.2871 & 0.1713 \\ \hline 1.3888 & -1.4322 & 1.1212 & -0.8335 & 0.0000\end{array}\right]$

\section{Appendix D: Controllers for Noncollocated} Two-Mass System

Linear quadratic Gaussian:

$\left[\begin{array}{rrrr|r}0.3077 & 1.7122 & 0.0012 & -0.0273 & -0.6525 \\ -0.7089 & -2.2107 & -0.0014 & 0.0316 & 0.7543 \\ 0.1117 & 0.4107 & -0.0103 & 1.4322 & -0.0000 \\ -3.1174 & -11.4642 & -1.4322 & -0.0103 & -0.0000 \\ \hline-2.6215 & -9.6403 & 0.0000 & -0.0000 & 0.0000\end{array}\right]$

Scaled Popov with $\gamma=15$ :

$\left[\begin{array}{rrrr|r}0.2047 & 1.6389 & -0.1243 & -0.0050 & -0.5692 \\ -0.6805 & -2.6373 & -0.0657 & 0.0478 & 0.6687 \\ 0.1126 & 0.4115 & -0.1831 & 1.4803 & 0.0394 \\ -3.0944 & -11.4614 & -1.5199 & -0.1868 & 0.0010 \\ \hline-2.5934 & -9.6228 & 0.1072 & -0.2978 & 0.0000\end{array}\right]$

Scaled Popov with $\gamma=4$ :

$\left[\begin{array}{rrrr|r}0.2579 & 1.6139 & -0.4487 & -0.1016 & -0.3647 \\ -0.7378 & -2.8287 & -0.1025 & -0.2842 & 0.1810 \\ 0.2062 & 0.3518 & -0.6588 & 1.7986 & -0.0788 \\ -3.1272 & -11.4576 & -1.7151 & -0.7245 & -0.2143 \\ \hline-2.5079 & -9.5863 & 0.3542 & 0.1859 & 0.0000\end{array}\right]$

Scaled Popov with $\gamma=2$ :

$\left[\begin{array}{rrrr|r}0.3594 & 1.5881 & -0.4578 & -0.1128 & -0.3585 \\ -0.7681 & -2.8158 & -0.1151 & -0.2956 & 0.0866 \\ 0.2285 & 0.3421 & -0.6905 & 1.8138 & 0.0544 \\ -3.1243 & -11.4611 & -1.7124 & -0.7469 & -0.3359 \\ \hline-2.4880 & -9.5837 & 0.3553 & 0.2164 & 0.0000\end{array}\right]$

Maximum entropy with $\delta_{2}=0.2$ :

$\left[\begin{array}{rrrr|r}0.2655 & 1.6802 & -0.0315 & -0.0196 & -0.6877 \\ -0.6794 & -2.3378 & -0.0133 & 0.0522 & 0.7758 \\ 0.1295 & 0.4054 & -0.0491 & 1.4531 & 0.0426 \\ -3.0704 & -11.4790 & -1.4465 & -0.0708 & -0.0675 \\ \hline-2.6677 & -9.6280 & 0.0146 & -0.0871 & 0.0000\end{array}\right]$

Maximum entropy with $\delta_{2}=0.5$ :

$\left[\begin{array}{rrrr|r}0.3804 & 1.5182 & -0.1677 & -0.0712 & -0.9465 \\ -0.8107 & -2.6761 & -0.0287 & 0.0783 & 0.8425 \\ 0.2708 & 0.3717 & -0.1846 & 1.6222 & 0.4701 \\ -2.7997 & -11.5855 & -1.4875 & -0.3952 & -0.4084 \\ \hline-2.9434 & -9.5884 & 0.0603 & -0.2656 & 0.0000\end{array}\right]$

Maximum entropy with $\delta_{2}=1.0$ :

$\left[\begin{array}{rrrr|r}0.3018 & 1.3856 & -0.4658 & -0.4377 & -0.8796 \\ -0.8640 & -3.4153 & -0.2048 & 0.0205 & 0.5153 \\ 0.4862 & 0.2629 & -0.4180 & 1.9616 & 0.7647 \\ -2.7855 & -11.5444 & -1.9418 & -1.2260 & -0.2143 \\ \hline-2.8143 & -9.7742 & 0.7496 & -0.2716 & 0.0000\end{array}\right]$

\section{References}

${ }^{1}$ Packard, A., and Doyle, J., "The Complex Structured Singular Value," Automatica, Vol. 29, 1993, pp. 71-109.

${ }^{2}$ Fan, M., Tits, A., and Doyle, J., "Robustness in the Presence of Mixed Parametric Uncertainty and Unmodeled Dynamics," IEEE Transactions on Automatic Control, Vol. 36, 1991, pp. 25-38.

${ }^{3}$ Haddad, W. M., and Bernstein, D. S., "Parameter-Dependent Lyapunov Functions and the Popov Criterion in Robust Analysis and Synthesis," IEEE Transactions on Automatic Control, Vol. 40, 1995, pp. 536-543.

${ }^{4}$ Bernstein, D. S., Haddad, W. M., and Sparks, A. G., "A Simplified Proof of the Popov Criterion and an Upper Bound for the Structured Singular Value with Real Parameter Uncertainty," Proceedings of the IEEE Conference on Decision and Control, Orlando, FL, 1994, pp. 2139,2140 .

${ }^{5}$ Sparks, A. G., and Bernstein, D. S., "The Scaled Popov Criterion and Bounds for the Real Structured Singular Value," Proceedings of the IEEE Conference on Decision and Control, Orlando, FL, 1994, pp. 2998-3002.

${ }^{6}$ Sparks, A. G., and Bernstein, D. S., "Reliable State Space Upper Bounds for the Peak Structured Singular Value," Proceedings of the American Control Conference, Seattle, WA, 1995.

${ }^{7}$ Friedman, J. H., and Bernstein, D. S., "Maximum Entropy Controller Synthesis for Collocated and Noncollocated Systems," Journal of Guidance, Control, and Dynamics, Vol. 17, 1993, pp. 859-862.

${ }^{8}$ How, J., Haddad, W., and Hall, S., "Application of Popov Controller Synthesis to Benchmark Problems with Real Parameter Uncertainty," Journal of Guidance, Control, and Dynamics, Vol. 17, No. 4, 1994, pp. 759-768.

${ }^{9}$ Haddad, W., How, J., Hall, S., and Bernstein, D. S., "Extensions of Mixed $\mu$ Bounds to Monotonic and Odd Monotonic Nonlinearities Using Absolute Stability Theory," International Journal of Control, Vol. 60, 1994, pp. 905-951.

${ }^{10}$ Kahaner, D., Moler, C., and Nash, S., Numerical Methods and Software, Prentice-Hall, Englewood Cliffs, NJ, 1988

${ }^{11}$ Dennis, I. E., and Schnabel, R. B., Numerical Methods for Unconstrained Optimization and Nonlinear Equations, Prentice-Hall, Englewood Cliffs, NJ, 1983.

${ }^{12}$ Wang, Y. W., and Bernstein, D. S., " $\mathrm{H}_{2}$ Optimal Control with an $\alpha-$ Shifted Pole Constraint," International Journal of Control, Vol. 58, 1993, pp. 1201-1214.

${ }^{13}$ Boyd, S., El Ghaoui, L., Feron, E., and Balakrishnan, V., Linear Matrix Inequalities in Systems and Control Theory, Society for Industrial and Applied Mathematics Studies in Applied Mathematics, Vol. 15, 1994.

${ }^{14}$ Bernstein, D. S., and Hyland, D. C., "Optimal Projection Approach to Fixed-Structure Control Design," Mechanics and Control of Large Flexible Structures, edited by J. L. Junkins, AIAA, Washington, DC, 1993, Chap. 10.

${ }^{15}$ Haddad, W. M., and Bernstein, D. S., "On the Gap Between $\mathrm{H}_{2}$ and Entropy Performance Measures in $\mathrm{H}_{\infty}$ Control," Systems and Control Letters, Vol. 14, 1990, pp. 113-120.

${ }^{16}$ Ridgely, D. B., Valavani, L., Dahleh, M., and Stein, G., "Solution to the General Mixed $\mathrm{H}_{2} / \mathrm{H}_{\infty}$ Control Problem-Necessary Conditions for Optimality," Proceedings of the American Control Conference, Chicago, IL, 1992, pp. 1348-1352. 